\title{
Post-exertional symptoms distinguish Myalgic Encephalomyelitis/Chronic Fatigue Syndrome subjects from healthy controls
}

\author{
Lariel J. Mateo ${ }^{\mathrm{a}}$, Lily Chu ${ }^{\mathrm{b}}$, Staci Stevens ${ }^{\mathrm{c}}$, Jared Stevens ${ }^{\mathrm{c}}$, Christopher R. Snell ${ }^{\mathrm{c}}$, \\ Todd Davenport ${ }^{\mathrm{a}, \mathrm{c}}$ and J. Mark VanNess ${ }^{\mathrm{a}, \mathrm{c} *}$ \\ ${ }^{a}$ University of the Pacific, Stockton, CA, USA \\ ${ }^{\mathrm{b}}$ Independent Consultant, Burlingame, CA, USA \\ ${ }^{\mathrm{c}}$ Workwell Foundation, Ripon, CA, USA
}

\begin{abstract}
.
BACKGROUND: Post-exertional malaise (PEM) is an exacerbation of symptoms that leads to a reduction in functionality. Recognition of PEM is important for the diagnosis and treatment of Myalgic Encephalomyelitis/Chronic Fatigue Syndrome (ME/CFS).

OBJECTIVE: Symptoms following cardiopulmonary exercise testing were compared between ME/CFS patients and healthy controls.

METHODS: Open-ended questionnaires were provided to subjects following two maximal exercise tests, 24 hours apart. Subjects evaluated how they felt at five time points. Responses were classified into 19 symptom categories.

RESULTS: ME/CFS subjects $(n=49)$ reported an average of $14 \pm 7$ symptoms compared to $4 \pm 3$ by controls $(n=10)$. During the seven days afterwards, ME/CFS subjects reported $4 \pm 3$ symptoms. None were reported by controls. Fatigue, cognitive dysfunction, and sleep problems were reported with the greatest frequency. ME/CFS patients reported more symptom categories at higher frequencies than controls. The largest differences were observed in cognitive dysfunction, decrease in function, and positive feelings.
\end{abstract}

CONCLUSIONS: A standardized exertional stimulus produced prolonged, diverse symptoms in ME/CFS subjects. This provides clues to the underlying pathophysiology of ME/CFS, leading to improved diagnosis and treatment.

Keywords: Post-exertional malaise, cardiopulmonary exercise test, symptoms, ME/CFS

\section{Introduction}

Patients with Myalgic Encephalomyelitis/Chronic Fatigue Syndrome (ME/CFS) experience symptoms, such as fatigue, sleep disturbances, problems think-

*Address for correspondence: J. Mark VanNess, Ph.D., Dept of Health and Exercise Science, University of the Pacific, Stockton, CA 95211, USA. E-mail: mvanness@ pacific.edu. ing, musculoskeletal pain and headache as well as symptoms related to gastrointestinal, cardiopulmonary, and immunological function [1-5]. The onset and persistence of such symptoms can produce a lasting and debilitating condition [1, 4-7]. According to the United States' Centers for Disease Control and Prevention (CDC), between 1 and 2.5 million people in the United States have reported symptoms associated with ME/CFS, yet approximately 84 per- 
cent have not been diagnosed [4, 7]. Women are estimated to be affected at 3 to 4 times the rate of men, with the disease often beginning during teenage years or between the ages of 30 and 39 [8].

One of the hallmark symptoms of ME/CFS is postexertional malaise (PEM) [1, 2, 4, 5, 7-12]. The phenomenon was first described by Ramsay in 1986 as a prolonged recovery from minimal exertion [13, 14]. The definition ensued from his experiences during the Royal Free London Outbreak in 1955 [13, 14]. In 1988, without actual use of the term, Holmes proposed that prolonged fatigue after previously tolerated levels of exercise was a common experience among ME/CFS patients [15, 16]. The term, PEM, was first introduced by the US CDC in 1994 as part of the Fukuda Criteria, but the symptom was not required for the diagnosis of ME/CFS [16]. It was not until 2003 that PEM, along with the inability to perform activities of daily living and unrefreshing sleep, were made mandatory criteria for diagnosis by the Canadian Consensus Criteria (CCC) [1]. In 2015, the United States' National Academy of Medicine (NAM) was tasked with updating the clinical diagnostic criteria for ME/CFS based on the cumulative scientific literature, patient accounts, and clinician experiences [17]. This new criteria not only required PEM but highlighted it as a key feature of the condition [17]. Consequently, the CDC has recommended that henceforth, clinicians diagnose using the NAM criteria, whereas the National Institutes of Health has requested that researchers employ either the NAM or CCC criteria [7, 17]. Therefore, a comprehensive, accurate understanding of PEM is more vital and urgent than ever.

PEM is currently characterized by an exacerbation of symptoms following physiological (movement or orthostatic/neuromuscular) stress and/or cognitive activity that the patient once engaged in without consequence $[1,4,9,12,16]$. PEM decreases functional ability and forces individuals with ME/CFS to restrict, reduce, and/or modify activities to avoid suffering amplified symptoms [4, 16, 19]. For example, mildly-affected patients may have to sit down to cook rather than stand up, as the latter requires more energy. More severely affected patients may have to rely on others to prepare meals entirely. It is often assumed that PEM is caused by physical deconditioning and that inactivity is a learned response [12]. However, exercise-based programs exclusively designed to improve fitness have been deemed ineffective and may be detrimental to ME/CFS patients [12]. Conversely, the Energy Envelope Theory by
Jason and the concept of pacing both of which describe the regulation of activity in proportion to exertional limits have been shown to increase activity and manage symptoms associated with PEM [12]. Ramsay previously encouraged patients to determine their exertional thresholds and alter their lifestyle patterns accordingly [12].

Several studies have revealed cardiovascular, immunological, neurological, metabolic and genetic expression abnormalities during or after physical activity in ME/CFS patients $[1,5,15,16,18]$. However, determination of the pathophysiology behind PEM remains difficult, as the triggers, onset, duration, intensity and symptoms of PEM can vary among patients $[4,7,10,15,16,19,20]$. Ordinary activities, such as taking a shower, dressing, reading the newspaper, watching a movie, climbing stairs, running errands and cleaning the house, can trigger the onset of PEM in some patients, while slightly more strenuous activities are needed to trigger the onset in others $[10,16,19]$. Symptoms of various magnitudes can start immediately or be delayed by hours or days, and may last for a few days, weeks, or even months $[15,16]$. Additionally, the experience reported by a ME/CFS patient during one episode of PEM may differ from that reported during a future episode [10, $16]$.

Although PEM is considered a cardinal symptom of ME/CFS, its reliability as a criterion for diagnosis is weakened by its subjective and variable presentation $[2,4,7,9,12,16]$.Issues encountered during past studies examining the phenomenon include classifying the symptoms that define PEM and adequately describing the experiences of ME/CFS patients [4]. As a result, few studies have investigated PEM from the patient perspective. The study by VanNess et al. [5] and the paper by Chu [11] analyzed self-reports from ME/CFS subjects after exertional triggers to define PEM. The work by VanNess and colleagues [5] also demonstrated symptoms following two-day cardiopulmonary exercise testing (CPET), a procedure often used for disability evaluation. While useful, these studies took place in a small group of subjects or did not include a control group [4, 5].

Occupational therapists (OTs) play a vital role in assisting patients to maximize function despite their health limitations. We suspect that OTs currently take care of ME/CFS, although given the high rate of underdiagnosis, these patients may be referred with vague diagnoses like "chronic fatigue" or "deconditioning." OTs who are able to recognize PEM may provide the patient and referring clinician with 
the specific diagnosis of ME/CFS. Alternatively, the patient may be diagnosed accurately but the OT may be unfamiliar with how to manage ME/CFS symptoms. These patients may not respond favorably to efforts to increase their activity. Furthermore, the decades-long standard of care, graded exercise therapy (GET) and cognitive behavioral therapy (CBT) have been retracted by health agencies like the CDC. Accumulating physiological studies, clinician experience, patient surveys, and re-analyses of trial results show that these treatments may not be helpful and may even be harmful for ME/CFS patients [23]. Incorporating information about PEM into patient care plans will increase effectiveness and patient acceptability while decreasing the chance of harm.

The purpose of the present study is to compare descriptions of symptoms by ME/CFS patients and control subjects after two maximal cardiopulmonary exercise tests, separated by 24 hours. The serial exercise test paradigm employs a controlled and standardized stimulus to induce PEM [20]. The subjects' descriptions from standardized, open-ended questionnaires serve to document symptoms of PEM over time and to determine whether PEM is unique to ME/CFS. It was hypothesized that symptom type, timing, and duration following exertional stress would be quantitatively and qualitatively different in ME/CFS patients compared to healthy controls and that findings would agree with those of prior studies.

\section{Materials and methods}

\subsection{Participants}

Sick subjects from different parts of the United States were referred by their health care providers to the testing facility for evaluation of functional capacity. Fifteen of the 49 were formally recruited for the study and met the Fukuda (1994) criteria. The remaining 34 were physician diagnosed. Ten sedentary, but otherwise healthy, control subjects from the community were also recruited for the study. Control subjects did not participate in an exercise program or perform more than 30 minutes of moderate physical activity on a weekly basis. All subjects were required to undergo and complete two days of exercise testing for inclusion in the study.

All participants signed an informed consent document prior to testing. The study was reviewed and approved by the Institutional Review Board at University of the Pacific.

\subsection{Two-day cardiopulmonary exercise testing}

Subjects were asked to refrain from all forms of physical activity for at least 24 hours before exercise testing. All protocols followed the procedures outlined by Stevens et al. [21] and described elsewhere $[2,19]$.

\subsection{Questionnaires}

Subjects were given an open-ended questionnaire to complete over the two days of testing and into the week following both tests. Subjects evaluated how they felt immediately after the first exercise test, before and immediately after the second exercise test, 24 hours after the second test and during the seven days after both tests. They were also asked to specify how long it took them to recover from the two-day CPET. For this, they chose from a range of answers (e.g., less than 1 day, 2 days, 7 days, still not recovered, etc.). Completed questionnaires were either mailed, faxed or emailed to the testing facility seven days after the second exercise test.

\subsection{Categorization of responses}

Fifty-nine surveys were digitized and labeled by study identification numbers. A coding schema, based on a review of literature and the authors' clinical and research experiences, was developed prior to the evaluation of the questionnaires. For example, the symptom category, 'Cognitive Dysfunction,' was designated when responses were related to memory, concentration, confusion and/or problems with communication, reading or information recall. Common jargon utilized by patients, such as "brain fog", "trouble finding words", or "feeling [mentally] out of it", were also considered acceptable descriptors for Cognitive Dysfunction. Next, researchers who were blinded to demographic information and subject diagnoses (LJM, LC) tested the schema on a small, random subset of surveys. The schema was then refined reiteratively to assure that it thoroughly and accurately reflected the experiences of subjects.

A total of nineteen codes, or symptom categories, were established. The symptom categories were Cardiopulmonary, Cognitive Dysfunction, Cold Limbs, Decrease in Function, Fatigue, Flu-like Symptoms, Gastrointestinal, Headache, Increase in Sensitivity, Light-headedness, Mood, Muscle/Joint Pain, Neurologic, Pain (for general pain or pain unfit for the other categories), Sleep Disturbances, Temperature 
Control, Tingling, Positive Feelings and Weakness. Excluding Positive Feelings, the remaining eighteen symptom categories were considered adverse. Representative keywords and phrases followed each category to classify responses. Two of the researchers (LJM and LC) independently reviewed each subjects' questionnaire using Excel spreadsheets which listed the nineteen symptom categories at each of the five time points (i.e., immediately after the first exercise test, 24 hours after the first exercise test, immediately after the second test, 24 hours after the second test and a week after the second test). Subjects' openended, written responses were interpreted as much as possible at face value, without effort to uncover the meaning behind phrases beyond the words written (i.e., reviewers focused on manifest rather than latent content). Spreadsheets were completed in a binary fashion. A ' 1 ' signified that the subject had experienced the symptom category at that time point and a ' 0 ' signified that the subject had not.

Upon completion of coding for a select number of questionnaires, the reviewers' results were compared to identify any differences. Where there were discrepancies, the reviewers referred to the original classification schema and arrived at a consensus for how to designate a response. The average discrepancy rate was $1.7 \%$ which equates to an average of roughly two differences within each survey. Four rounds of coding were necessary to review the fiftynine questionnaires (i.e., reviewers conducted the coding process for 16 questionnaires, 14 questionnaires, 13 questionnaires then 16 ).

\subsection{Data analysis}

All data were exported and organized into a master Microsoft Excel spreadsheet. The master spreadsheet included each subject's identification number, demographic and diagnostic data, the symptom categories at each time point, the final versions of the questionnaire interpretations and a column that determined whether the subject experienced the symptom category at any point. Total number, means, and standard deviations of symptom categories and duration of symptoms for both groups were calculated by a third researcher (JMV). Student's $t$-test was used to compare continuous data from each group, while Fisher's exact test compared categorical data (https://www.graphpad.com/quickcalcs/). Bonferroni's correction adjusted for multiple comparisons. A two-tailed $p$-value equal to or less than 0.0025 was considered significant.

\section{Results}

\subsection{Demographic analysis}

A total of 59 subjects participated in the study $(n=59)$. There were no statistical differences in age or anthropometric indices between the ME/CFS group and the control group. Seventy percent of the control and $80 \%$ of the ME/CFS subjects were female. The average age for the ME/CFS patients was $41.3 \pm 7.0$ years, while the average age for the control subjects was $35.3 \pm 12.2$ years. The average height and weight were $1.6 \pm 0.6 \mathrm{~m}$ and $65.5 \pm 10.3 \mathrm{~kg}$ for the ME/CFS group and $1.6 \pm 0.5 \mathrm{~m}$ and $68.7 \pm 9.5 \mathrm{~kg}$ for the control group.

\subsection{Quantitative analysis}

The post-exertional symptoms reported by the ME/CFS patients were more diverse than those reported by the control subjects. All symptom categories were reported by at least one subject from the ME/CFS group, except for the symptom category, Cold Limbs. Subjects in the control group did not report any symptoms corresponding to Cognitive Dysfunction, Decrease in Function, Flu-like Symptoms, Cold Limbs or Cardiopulmonary. On average, the ME/CFS subjects' responses accounted for more symptom categories than the control subjects' $(7.63 \pm 3.30$ vs. $1.90 \pm 1.52, p<0.001)$ (Table 1 , column 2). Over the days of testing, the average number of symptoms reported by the ME/CFS group was greater than that reported by the control group $(14.35 \pm 7.30$ vs. $3.50 \pm 2.59, p<0.002)$ (Table 1 , column 3). Adverse responses in the week following the two-day CPET were seen only in the ME/CFS group ( $4.14 \pm 2.65)$, with none of the control subjects reporting any symptoms (Table 1 , column 4 ).

Of the symptom categories that the control subjects experienced, the ME/CFS group reported almost all of them at greater frequencies at each of the five time points. Figure 1 displays eleven of the nineteen categories and the percentages at which the ME/CFS and control subjects reported them. The symptom categories are shown on the x-axis, while the associated percentages at each time points are shown on the y-axis. The symptom categories that were reported with the greatest frequency by the ME/CFS group are shown at the top of Fig. 1. These include Fatigue, Muscle/Joint Pain, Cognitive Dysfunction, Decrease in Function and Headache. For comparison, the symptom categories and their 
Table 1

Average number of symptom categories and symptoms during the two-day cardiopulmonary exercise test and during the week after the CPETs; and days to recover in the Myalgic Encephalomyelitis/Chronic Fatigue Syndrome (ME/CFS) and the control groups

\begin{tabular}{lcccc}
\hline Group & Symptom Categories & Two-day CPET & Thereafter & Days to Recover \\
\hline ME/CFS & $7.63(3.30)^{*}$ & $14.35(7.30)$ & $4.14(2.65)$ & 4.5 days** \\
Control & $1.90(1.52)^{*}$ & $3.50(2.59)$ & - & 1 day \\
\hline
\end{tabular}

*Out of 19 symptom categories ( 18 adverse, 1 positive). ${ }^{* *}$ Average number of days to recover based on data from 24 out of $49 \mathrm{ME} / \mathrm{CFS}$ subjects; the remaining 25 subjects took more than 7 days to recover.

percentages for the control group are seen at the bottom of Fig. 1. Similarly, Sleep Disturbances, Pain, Weakness, Cardiopulmonary, Light-headedness and Flu-like Symptoms are displayed for the ME/CFS and healthy groups at the top and bottom of Fig. 2, respectively. The only symptom categories with similar percentages between the ME/CFS subjects and the control subjects were 'Pain' at time point 1 (immediately after the first test, $16.3 \%$ vs. $10.0 \%$, respectively) and 'Weakness' at time points 2 (24 hours after the first test, $12.2 \%$ vs. $10.0 \%$ ) and 3 (immediately after the second test, $20.4 \%$ vs. $20.0 \%$ ). Table 2 shows symptom expression over time for further comparison. For each symptom category at each time point, counts and percentages are given for each group.

To account for subjects who might have reported the same symptoms during multiple time points (e.g., a single subject reporting Pain over three time periods vs. three different subjects each reporting Pain once during different time periods), Table 3 exhibits the number of subjects in each group who endorsed a symptom category during any of the five time periods. Except for Positive Feelings, all symptom categories were endorsed by more ME/CFS subjects than control subjects. Significantly more ME/CFS subjects experienced Cognitive Dysfunction and Decrease in Function than control subjects $(61 \%$ more subjects for both categories, $p<0.0025)$. Conversely, 52\% more control subjects communicated Positive Feelings than ME/CFS subjects $(p<0.0025)$.

The time to recover from the bouts of exercise was also dramatically different between the groups. For 24 of the $49 \mathrm{ME} / \mathrm{CFS}$ subjects, the average time to return to their baseline of health was 4.5 days (Table 1, column 5). The remaining $25 \mathrm{ME} / \mathrm{CFS}$ subjects reported that they had not recovered by the seventh day after the second exercise test. For the control group, recovery was rapid. All but one control subject returned to baseline in less than one day of the CPET (Table 1, column 5).

\subsection{Qualitative analysis}

The descriptions of symptoms were qualitatively different between the control and ME/CFS subjects. Control subjects used terse descriptions such as "fatigued," "tired," "headache," "sore," "hot" and "muscles hurt" to explain how they felt after the exercise tests. In contrast, ME/CFS subjects provided detailed accounts of their symptoms. One subject wrote:

"[I was] too fatigued to do any basic activities of daily living. I would have been in bed all day, but I was too weak/fatigued to walk back upstairs to get there, laid on couch all day instead. I have been on a downward spiral since the test. I have done nothing but rest, and I continue to feel worse and worse. The level of fatigue is indescribable. I've been too fatigued to shower for the last 4 days, to wash my hair since before the test, to make food, to answer phone, understand what I'm watching on TV, couldn't get my taxes done. This is the most extreme my fatigue has been in a long time."

Other typical statements from ME/CFS subjects about their experiences included:

"[Feeling] physically drained, mentally spent. Foggy sensation, overall weary."

"[Felt] like I was in a barrel and rolled down hill!"

"Felt like I'd been through trauma."

"I feel like I've been hit by a Mack truck."

"All week, I just couldn't seem to find enough energy to "get going" for the day."

"Trouble holding head and body up: had to recline as much as possible."

"Terrible headaches - felt like someone had struck the back of my neck with a baseball bat."

Many of the control subjects expressed positive feelings after exercise compared to ME/CFS sub- 

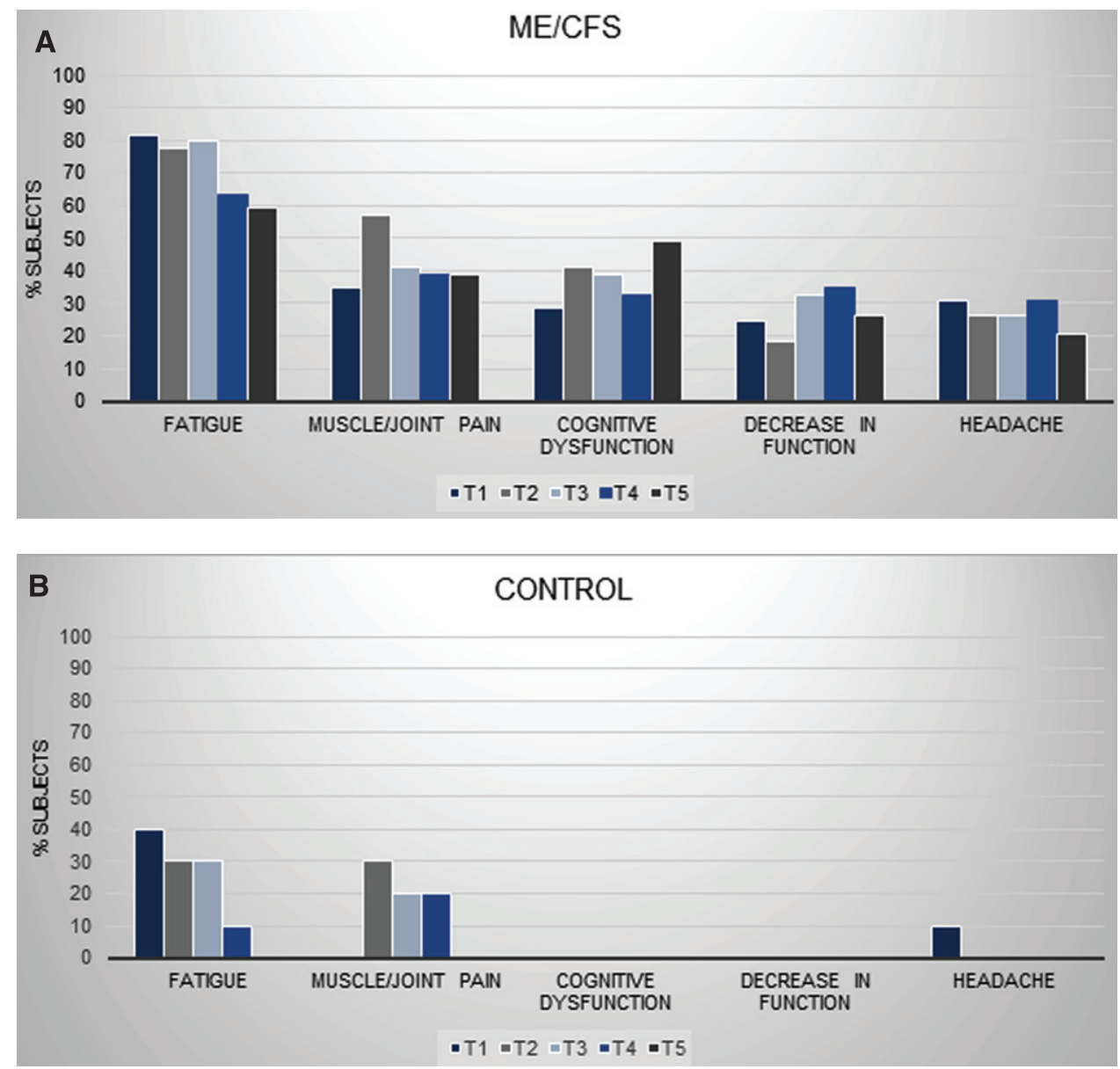

Fig. 1. Group comparisons for symptoms: Fatigue, Muscle/Joint Pain, Cognitive Function, Decrease in Function, and Headache.

jects. For example, two control subjects mentioned increased energy immediately afterwards which extended to the next day:

"Fine, went home and did housework. Actually felt a little energetic."

"Very energetic."

Collectively, the narrative of the post-exertional experience differs between an ME/CFS patient and a control subject.

\section{Discussion}

A standardized exertional stimulus produced prolonged, diverse symptoms in ME/CFS subjects. Although a few of the symptom categories were reported by both the ME/CFS patients and the control subjects, their post-exertional experiences were not comparable. The symptom categories the control group reported with the greatest frequency (i.e., Positive Feelings, Fatigue, Muscle/Joint Pain, and Weakness) are consistent with what individuals with similar, sedentary exercise habits might briefly experience following bouts of maximal exertion. Adverse symptoms appeared to be mild and may be explained by delayed onset muscle soreness [22]. Beneficial symptoms were likely stimulated by exercise-associated endorphins. As expected, once the exertional stimulus was removed, control subjects did not report negative symptoms of any kind and quickly returned to their baseline levels of health.

The occurrence, frequency, severity and duration of post-exertional symptoms in the ME/CFS group differed substantially from the healthy group. ME/CFS patients reported symptom categories not observed in the healthy controls and experienced 

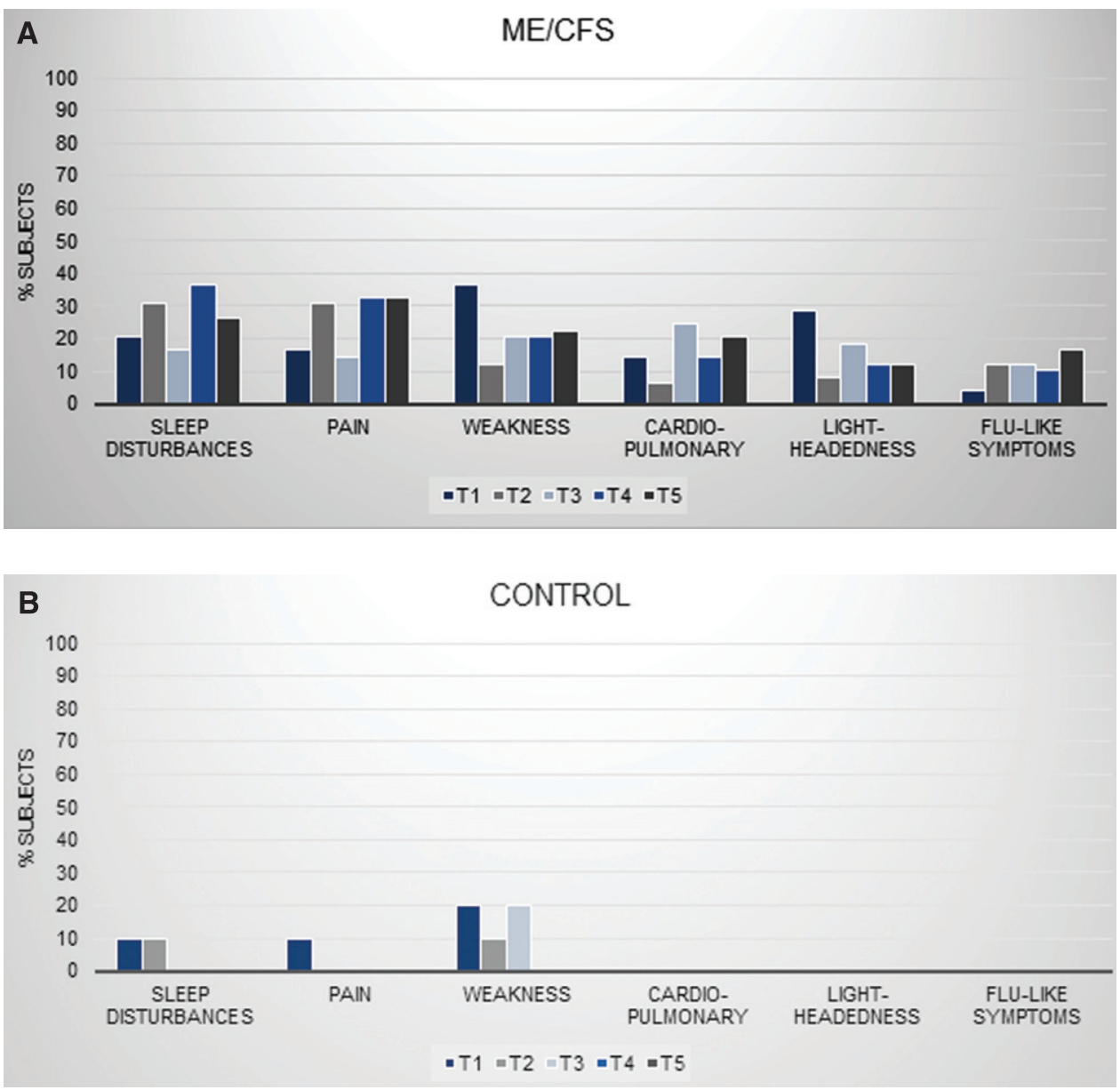

Fig. 2. Group comparisons for symptoms: Sleep Disturbances, Pain, Weakness, Cardiopulmonary, Light-headedness, and Flu-like Symptoms.

almost all shared symptom categories at a higher frequency, higher intensity, and for more days than the healthy controls. Only the ME/CFS group reported symptoms from the following categories: Cognitive Dysfunction, Decrease in Function, Cardiopulmonary Symptoms, Flu-like Symptoms, Gastrointestinal Disturbance, Mood Disturbance, Tingling, Neurologic Symptoms and Increase in Sensitivity. For the nine symptom categories that were experienced by both groups, $12 \%$ to $47 \%$ more ME/CFS subjects reported them, except for "Positive Feelings" which 52\% more healthy controls disclosed. Adverse symptoms significantly impacted subjects' lives and were endured for a median of at least 4.5 days.

The nineteen symptom categories and their corresponding descriptors are unique to this study. However, the post-exertional phenomena reported by the ME/CFS group match those reported in other studies and included in clinical definitions $[1,5,11]$.
ME/CFS patients commonly exhibit intense symptoms and poor recovery rates in response to exercise. In VanNess' study [5], for example, one fatigued control felt "fine overall" with only minor problems climbing stairs, whereas the ME/CFS counterpart could not walk without assistance following the exercise test. Only $4 \%$ to $9 \%$ of the ME/CFS subjects recovered entirely within 24 hours [5]. TD previously calculated that length of recovery could identify ME/CFS subjects with a positive likelihood ratio of 11.3 [2]. Overall, $85 \%$ of the healthy subjects reported feeling better post-exercise compared to $0 \%$ of the ME/CFS patients [5]. Furthermore, healthy control subjects did not experience any problems thinking or immunological symptoms (flu-like feelings, sore throat, or enlarged lymph nodes) following exercise in prior studies [4,9]. Author LC's survey of ME/CFS subjects revealed that approximately two-thirds of subjects noted cognitive issues or immunological 
Table 2

Symptom expression during serial cardiopulmonary exercise testing in individuals with ME/CFS and control subjects

\begin{tabular}{|c|c|c|c|c|c|c|}
\hline \multirow[t]{2}{*}{ Symptom } & \multirow[t]{2}{*}{ Group } & \multicolumn{2}{|c|}{ Test 1} & \multicolumn{3}{|c|}{ Test 2} \\
\hline & & Immediately & 24 hours & Immediately & 24 hours & 1 week \\
\hline \multirow[t]{2}{*}{ Fatigue } & ME/CFS & $40(81.6 \%)$ & $38(77.6 \%)$ & $39(79.6 \%)$ & $31(63.3 \%)$ & $29(59.2 \%)$ \\
\hline & Control & $4(40.0 \%)$ & $3(30.0 \%)$ & $3(30.0 \%)$ & $1(10.0 \%)$ & $0(0.0 \%)$ \\
\hline \multirow[t]{2}{*}{ Muscle/Joint Pain } & $\mathrm{ME} / \mathrm{CFS}$ & $17(34.7 \%)$ & $28(57.1 \%)$ & $20(40.8 \%)$ & $19(38.8 \%)$ & $19(38.8 \%)$ \\
\hline & Control & $0(0.0 \%)$ & $3(30.0 \%)$ & $2(20.0 \%)$ & $2(20.0 \%)$ & $0(0.0 \%)$ \\
\hline \multirow[t]{2}{*}{ Cognitive Dysfunction } & ME/CFS & $14(28.6 \%)$ & $20(40.8 \%)$ & $19(38.8 \%)$ & $16(32.7 \%)$ & $24(50.0 \%)$ \\
\hline & Control & $0(0.0 \%)$ & $0(0.0 \%)$ & $0(0.0 \%)$ & $0(0.0 \%)$ & $0(0.0 \%)$ \\
\hline \multirow[t]{2}{*}{ Decrease in Function } & $\mathrm{ME} / \mathrm{CFS}$ & $12(24.5 \%)$ & $9(18.4 \%)$ & $16(32.7 \%)$ & $17(34.7 \%)$ & $13(26.5 \%)$ \\
\hline & Control & $0(0.0 \%)$ & $0(0.0 \%)$ & $0(0.0 \%)$ & $0(0.0 \%)$ & $0(0.0 \%)$ \\
\hline \multirow[t]{2}{*}{ Headache } & ME/CFS & $15(30.6 \%)$ & $13(26.5 \%)$ & $13(26.5 \%)$ & $15(30.6 \%)$ & $10(20.4 \%)$ \\
\hline & Control & $1(10.0 \%)$ & $0(0.0 \%)$ & $0(0.0 \%)$ & $0(0.0 \%)$ & $0(0.0 \%)$ \\
\hline \multirow[t]{2}{*}{ Sleep Disturbances } & $\mathrm{ME} / \mathrm{CFS}$ & $10(20.4 \%)$ & $15(30.6 \%)$ & $8(16.3 \%)$ & $18(36.7 \%)$ & $13(26.5 \%)$ \\
\hline & Control & $1(10.0 \%)$ & $1(10.0 \%)$ & $0(0.0 \%)$ & $0(0.0 \%)$ & $0(0.0 \%)$ \\
\hline \multirow[t]{2}{*}{ Pain } & $\mathrm{ME} / \mathrm{CFS}$ & $8(16.3 \%)$ & $15(30.6 \%)$ & $7(14.3 \%)$ & $16(32.7 \%)$ & $16(32.7 \%)$ \\
\hline & Control & $1(10.0 \%)$ & $0(0.0 \%)$ & $0(0.0 \%)$ & $0(0.0 \%)$ & $0(0.0 \%)$ \\
\hline \multirow[t]{2}{*}{ Weakness } & $\mathrm{ME} / \mathrm{CFS}$ & $18(36.7 \%)$ & $6(12.2 \%)$ & $10(20.4 \%)$ & $10(20.4 \%)$ & $11(22.4 \%)$ \\
\hline & Control & $2(20.0 \%)$ & $1(10.0 \%)$ & $2(20.0 \%)$ & $0(0.0 \%)$ & $0(0.0 \%)$ \\
\hline \multirow[t]{2}{*}{ Cardiopulmonary Symptoms } & $\mathrm{ME} / \mathrm{CFS}$ & $7(14.3 \%)$ & $3(6.1 \%)$ & $12(24.5 \%)$ & $7(14.3 \%)$ & $10(20.4 \%)$ \\
\hline & Control & $0(0.0 \%)$ & $0(0.0 \%)$ & $0(0.0 \%)$ & $0(0.0 \%)$ & $0(0.0 \%)$ \\
\hline \multirow{2}{*}{ Light-headedness } & $\mathrm{ME} / \mathrm{CFS}$ & $14(28.6 \%)$ & $4(8.2 \%)$ & $9(18.4 \%)$ & $6(12.2 \%)$ & $6(12.2 \%)$ \\
\hline & Control & $1(10.0 \%)$ & $0(0.0 \%)$ & $0(0.0 \%)$ & $0(0.0 \%)$ & $0(0.0 \%)$ \\
\hline \multirow[t]{2}{*}{ Flu-like Symptoms } & $\mathrm{ME} / \mathrm{CFS}$ & $2(4.1 \%)$ & $6(12.2 \%)$ & $6(12.2 \%)$ & $5(10.2 \%)$ & $8(16.3 \%)$ \\
\hline & Control & $0(0.0 \%)$ & $0(0.0 \%)$ & $0(0.0 \%)$ & $0(0.0 \%)$ & $0(0.0 \%)$ \\
\hline \multirow[t]{2}{*}{ Gastrointestinal Disturbance } & $\mathrm{ME} / \mathrm{CFS}$ & $6(12.2 \%)$ & $3(6.1 \%)$ & $5(10.2 \%)$ & $6(12.2 \%)$ & $7(14.3 \%)$ \\
\hline & Control & $0(0.0 \%)$ & $0(0.0 \%)$ & $0(0.0 \%)$ & $0(0.0 \%)$ & $0(0.0 \%)$ \\
\hline \multirow[t]{2}{*}{ Mood Disturbance } & $\mathrm{ME} / \mathrm{CFS}$ & $2(4.1 \%)$ & $3(6.1 \%)$ & $7(14.3 \%)$ & $4(8.2 \%)$ & $6(12.2 \%)$ \\
\hline & Control & $0(0.0 \%)$ & $0(0.0 \%)$ & $0(0.0 \%)$ & $0(0.0 \%)$ & $0(0.0 \%)$ \\
\hline \multirow[t]{2}{*}{ Temperature Control } & $\mathrm{ME} / \mathrm{CFS}$ & $7(14.3 \%)$ & $1(2.0 \%)$ & $3(6.1 \%)$ & $4(8.2 \%)$ & $4(8.2 \%)$ \\
\hline & Control & $1(10.0 \%)$ & $0(0.0 \%)$ & $1(10.0 \%)$ & $0(0.0 \%)$ & $0(0.0 \%)$ \\
\hline \multirow{2}{*}{ Tingling } & $\mathrm{ME} / \mathrm{CFS}$ & $2(4.1 \%)$ & $2(4.1 \%)$ & $4(8.2 \%)$ & $2(4.1 \%)$ & $4(8.2 \%)$ \\
\hline & Control & $0(0.0 \%)$ & $0(0.0 \%)$ & $0(0.0 \%)$ & $0(0.0 \%)$ & $0(0.0 \%)$ \\
\hline \multirow[t]{2}{*}{ Neurologic Symptoms } & $\mathrm{ME} / \mathrm{CFS}$ & $1(2.0 \%)$ & $1(2.0 \%)$ & $1(2.0 \%)$ & $3(6.1 \%)$ & $4(8.2 \%)$ \\
\hline & Control & $0(0.0 \%)$ & $0(0.0 \%)$ & $0(0.0 \%)$ & $0(0.0 \%)$ & $0(0.0 \%)$ \\
\hline \multirow[t]{2}{*}{ Increase in Sensitivity } & $\mathrm{ME} / \mathrm{CFS}$ & $0(0.0 \%)$ & $2(4.1 \%)$ & $2(4.1 \%)$ & $1(2.0 \%)$ & $1(2.0 \%)$ \\
\hline & Control & $0(0.0 \%)$ & $0(0.0 \%)$ & $0(0.0 \%)$ & $0(0.0 \%)$ & $0(0.0 \%)$ \\
\hline \multirow[t]{2}{*}{ Cold Limbs } & $\mathrm{ME} / \mathrm{CFS}$ & $0(0.0 \%)$ & $0(0.0 \%)$ & $0(0.0 \%)$ & $0(0.0 \%)$ & $0(0.0 \%)$ \\
\hline & Control & $0(0.0 \%)$ & $0(0.0 \%)$ & $0(0.0 \%)$ & $0(0.0 \%)$ & $0(0.0 \%)$ \\
\hline \multirow[t]{2}{*}{ Positive Feelings } & $\mathrm{ME} / \mathrm{CFS}$ & $4(8.2 \%)$ & $5(10.2 \%)$ & $3(6.1 \%)$ & $3(6.1 \%)$ & $1(2.0 \%)$ \\
\hline & Control & $5(50.0 \%)$ & $6(60.0 \%)$ & $6(60.0 \%)$ & $7(70.0 \%)$ & $0(0.0 \%)$ \\
\hline
\end{tabular}

Time points include immediately after the first CPET, 24 hours after the first CPET, immediately after the second CPET, 24 hours after the second CPET, and 1 week after the second CPET. Parenthetical values are within-group symptom prevalence.

symptoms when they overexerted themselves in their daily lives [11]. Thus, exercising at absolute intensities has been shown to benefit healthy subjects but induce PEM in ME/CFS patients.

\subsection{Limitations}

There are several limitations in the study. First, the average age of the study sample is younger than in other ME/CFS studies. Second, the questionnaire did not quantify length of recovery beyond seven days, decreases in functionality, and symptom magnitude. There were options for subjects who recovered in less than one day and in 2 days, but not for subjects who recovered in 1 day. Subjects also could not dis- close how many days past a week it took them to return to their baseline level of health. Open-ended responses were restricted to a one-week timeframe following the CPET. Future questionnaires might include a standardized instrument that allows subjects to describe decrements in the ability to perform basic or instrumental activities of daily living. Furthermore, a study should be performed to assess and quantify the severity of reported symptoms. If post-exertional symptoms had been reported both quantitatively (i.e., numerical representations for symptom magnitude) and qualitatively (i.e., descriptions written by subjects) within the current survey, the data might have shown that the symptom categories reported by both subject groups were experienced at disparate inten- 
Table 3

Number and percentage of subjects in each group endorsing a symptom category during any of the 5 time periods

\begin{tabular}{|c|c|c|c|c|c|}
\hline Symptom Category & $\begin{array}{c}\text { Control }(\mathrm{N}) \\
(\mathrm{N}=10 \text { total })\end{array}$ & Control (\%) & $\begin{array}{l}\text { ME/CFS }(\mathrm{N}) \\
(\mathrm{N}=49 \text { total })\end{array}$ & ME/CFS (\%) & $\%$ Difference $^{1}$ \\
\hline Fatigue & 6 & 60 & 47 & 96 & 36 \\
\hline Muscle/Joint Pain & 4 & 40 & 40 & 82 & 42 \\
\hline Cognitive Dysfunction & 0 & 0 & 30 & 61 & $61^{2}$ \\
\hline Decrease in Function & 0 & 0 & 30 & 61 & $61^{2}$ \\
\hline Headache & 1 & 10 & 28 & 57 & 47 \\
\hline Sleep Disturbances & 1 & 10 & 28 & 57 & 47 \\
\hline Pain & 1 & 10 & 26 & 53 & 43 \\
\hline Weakness & 2 & 20 & 27 & 55 & 35 \\
\hline Cardiopulmonary Symptoms & 0 & 0 & 19 & 39 & 39 \\
\hline Light-headedness & 1 & 10 & 23 & 47 & 37 \\
\hline Flu-like Symptoms & 0 & 0 & 13 & 27 & 27 \\
\hline Gastrointestinal Disturbance & 0 & 0 & 14 & 29 & 29 \\
\hline Mood Disturbance & 0 & 0 & 13 & 29 & 29 \\
\hline Temperature Control & 1 & 10 & 11 & 22 & 12 \\
\hline Tingling & 0 & 0 & 5 & 10 & 10 \\
\hline Neurologic Symptoms & 0 & 0 & 7 & 14 & 14 \\
\hline Increase in Sensitivity & 0 & 0 & 3 & 6 & 6 \\
\hline Cold Limbs & 0 & 0 & 0 & 0 & 0 \\
\hline Positive Feelings & 7 & 70 & 9 & 18 & $-52^{2}$ \\
\hline
\end{tabular}

${ }^{1}$ This is the difference in percentages of subjects experiencing a symptom category. Except for "Positive feelings" which were cited more frequently by Control subjects, a higher percentage of ME/CFS subjects suffered from each symptom category. ${ }^{2} p$-values less than 0.0025 .

sities. Third, the coding schema was constructed based on our ideas about PEM. The independent reviewers used it to categorize responses at face value. Latent content analysis and in-depth questioning might reveal further details or lead to different interpretations. Finally, our findings are based on subjects' responses after maximal effort and physical exertion. Submaximal or other types of stressors may produce different results. Despite these limitations, these results agree with and support prior studies. Future studies may consider designing a study without these limitations and including objective CPET results within the analysis.

\section{Conclusion}

The presence of symptoms during and after the two-day CPET and the extended recovery time in the ME/CFS group exemplify PEM. Two key symptoms, cognitive dysfunction and a decrease in function, can potentially indicate the occurrence of PEM. This is consistent with a 2019 suvey of 1,534 ME/CFS subjects where the top selected PEM consequence was "reduced stamina and functional ability" (selected by $99.4 \%$ ) followed by "physical fatigue" (98.9\%), "cognitive exhaustion" (97.4\%), and "problems thinking" (97.4\%). Other complaints prominent in ME/CFS patients after activity are fatigue, muscle/joint pain, headaches and sleep disturbances. The disparity in the time to recover may serve as an additional marker. These symptoms and the rate of recovery contribute to the existing literature on PEM and assist health care professionals in recognizing PEM. By discerning both the attributes of PEM and its manifestations in each patient, health care providers can identify patients with ME/CFS and devise patient-specific treatment plans to combat the onset and reduce the severity of symptoms. OTs are already familiar with the concepts of energy conservation and activity management but these concepts may need to be modified for ME/CFS patients. For example, more rest may be needed following activity compared to other medical conditions. Patients may reach a plateau of activity which is improved but not close to "normal" since the physiological underpinnings of ME/CFS are not yet fully understood. Early diagnosis and symptom management promote the maintenance of or even improvement in patient function.

This paper also sheds light on the possible risks of a 2-day CPET. It does not appear that patients or research subjects suffer permanent or protracted damage from CPET. Our data show that recovery is bimodal, with approximately half of subjects taking a week or less to recover. This information can 
be shared with patients or research subjects so that they might make a betterinformed decision. Exploration of this bimodal pattern may also help discover subgroups within or the pathophysiology underlying ME/CFS.

\section{Acknowledgments}

We thank the subjects in this study for volunteering their time and energy to participate in this project and contribute to the findings.

\section{Conflict of interest}

None declared.

\section{References}

[1] Carruthers BM, Jain AK, De Meirleir KL. Myalgic encephalomyelitis/chronic fatigue syndrome: A clinical case definition and guidelines for medical practitioners. Journal of Chronic Fatigue Syndrome. 2003;11(1):7-115.

[2] Davenport TE, Stevens SR, Baroni K, VanNess M, Snell CR. Diagnostic accuracy of symptoms characterizing chronic fatigue syndrome. Disability and Rehabilitation. 2011;33:19-20.

[3] Davenport TE, Stevens SR, Baroni K, VanNess M, Snell CR. Reliability and validity of Short Form 36 Version 2 to measure health perceptions in a sub-group of individuals with fatigue. Disability and Rehabilitation. 2011;33:25-26.

[4] U.S. Food and Drug Administration (FDA). The Voice of the Patient: Chronic Fatigue Syndrome and Myalgic Encephalomyelitis; 2013. Available from https://www.fda. gov/downloads/forindustry/userfees/prescriptiondruguser fee/ucm368806.pdf.

[5] Van Ness JM, Stevens SR, Bateman L, Stiles TL, Snell CR. Postexertional malaise in women with chronic fatigue syndrome. J Womens Health (Larchmt). 2010;19(2):239-244.

[6] Ciccolella ME, Davenport TE. Scientific and legal challenges to the functional capacity evaluation in chronic fatigue syndrome. Fatigue: Biomedicine, Health \& Behavior. 2013;1(4):243-55.

[7] Committee on the Diagnostic Criteria for Myalgic Encephalomyelitis/Chronic Fatigue Syndrome, Board on the Health of Select Populations, Institute of Medicine. Beyond myalgic encephalomyelitis/chronic fatigue syndrome: Redefining an illness. Washington (DC): National Academies Press; 2015.
[8] Bakken IJ, Tveito K, Gunnes N, Ghaderi S, Stoltenberg C, Trogstad L, Haberg SE, Magnus P. Two age peaks in the incidence of chronic fatigue syndrome/myalgic encephalomyelitis: A population-based registry study from Norway 2008-2012. BMC Med. 2014;12:1-167.

[9] Jason LA, Evans M, So S, Scott J, Brown A. Problems in defining post-exertional malaise. Journal of Prevention \& Intervention in the Community. 2015;43(1):20-31.

[10] McManimen SL, Jason LA. Differences in me and cfs symptomology in patients with normal and abnormal exercise test results. Int J Neurol Neurother. 2017;4(1):1-7.

[11] Chu L, Valencia IJ, Garvert DW, Montoya JG. Deconstructing post-exertional malaise in myalgic encephalomyelitis/ chronic fatigue syndrome: A patient-centered, crosssectional survey. Plos One. 2018;13(6):e0197811.

[12] Goudsmit EM, Nijs J, Jason LA, Wallman KE. Pacing as a strategy to improve energy management in myalgic encephalomyelitis/chronic fatigue syndrome: A consensus document. Disabil Rehabil. 2012;34(13):1140-47.

[13] Ramsay AM. Myalgic encephalomyelitis: A baffling syndrome with a tragic aftermath. M.E. Association Journal. 1989;1-3.

[14] Ramsay AM. Myalgic encephalomyelitis and postviral fatigue states: the saga of royal free disease. United Kingdom: Gower Medical Publishing. 1986.

[15] Holmes GP, Kaplan JE, Gantz NM. Chronic fatigue syndrome: A working case definition. Ann Intern Med. 1988;108:387-9.

[16] Spotila J. Post-exertional malaise in chronic fatigue syndrome. CFIDS Association of America. 2010;1-12

[17] National Academy of Medicine. IOM 2015 diagnostic criteria; 2018. Available from: https://www.cdc.gov/mecfs/healthcare-providers/diagnosis/iom-2015-diagnosticcriteria.html/.

[18] Carruthers BM, van de Sande MI, De Meirleir KL. Myalgic encephalomyelitis: International consensus criteria. Journal of Internal Medicine. 2011;270:327-38.

[19] VanNess JM, Snell CR, Strayer DR, Dempsey L 4th, Stevens SR. Subclassifying chronic fatigue syndrome through exercise testing. Med Sci Sports Exerc. 2003;35(6):908-13.

[20] Hodges LD, Nielsen T, Baken D. Physiological measures in participants with chronic fatigue syndrome, multiple sclerosis and healthy controls following repeated exercise: A pilot study. Clin Physiol Funct Imaging. 2017.

[21] Stevens S, Snell C, Stevens J, Keller B, VanNess JM. Cardiopulmonary exercise test methodology for assessing exertion intolerance in myalgic encephalomyelitis/chronic fatigue syndrome. Front. Pediatr. 2018;6:242.

[22] Contro V, Mancuso EP, Proia P. Delayed onset muscle soreness (DOMS) management: Present state of the art. Trends in Sport Sciences. 2016;23(3):121-27.

[23] Wilshire CE, Kindlon T, Courtney R, Matthees A, Tuller $\mathrm{D}$, Geraghty K, et al. Rethinking the treatment of chronic fatigue syndrome - a reanalysis and evaluation of findings from a recent major trial of graded exercise and CBT. BMC Psychol. 2018;6:6. 


\section{Appendix: Questionnaire used for collection of reported symptoms}

\section{Exercise Recovery Questionnaire}

Study ID: Date of Exercise Tests:

1. How did you feel following the first exercise test?

2. Describe how you felt the day after the first exercise test.

3. How did you feel following the second exercise test?

4. Describe how you felt the day after the second exercise test.

5. How long did it take you to recover from the exercise tests? Circle the appropriate time...(in days) less than 1234567 still not recovered

Comments:

6. Describe symptoms, if any, experienced after the exercise tests. 